\title{
Amino acid-insensitive mTORC1 regulation enables nutritional stress resilience in hematopoietic stem cells
}

\author{
Demetrios Kalaitzidis, ${ }^{1,2,3,4}$ Dongjun Lee, ${ }^{1,2,3,4}$ Alejo Efeyan, ${ }^{5,6,7,8,9}$ Youmna Kfoury, ${ }^{1,2,3,4}$ Naema Nayyar, ${ }^{1,2,8}$ David B. Sykes, ${ }^{1,2,3,4}$ \\ Francois E. Mercier, ${ }^{1,2,3,4}$ Ani Papazian, ${ }^{1,2,3,4}$ Ninib Baryawno, ${ }^{1,2,3,4}$ Gabriel D. Victora, ${ }^{5}$ Donna Neuberg, ${ }^{10}$ David M. Sabatini,, ${ }^{5,6,7,8,9}$ \\ and David T. Scadden ${ }^{1,2,3,4}$ \\ ${ }^{1}$ Center for Regenerative Medicine and ${ }^{2}$ Cancer Center, Massachusetts General Hospital, Boston, Massachusetts, USA. ${ }^{3}$ Department of Stem Cell and Regenerative Biology and ${ }^{4}$ Harvard Stem Cell Institute, \\ Harvard University, Cambridge, Massachusetts, USA. ${ }^{5}$ Whitehead Institute for Biomedical Research, Cambridge, Massachusetts, USA. ${ }^{6}$ Department of Biology and ${ }^{7}$ Howard Hughes Medical Institute, \\ Massachusetts Institute of Technology, Cambridge, Massachusetts, USA. ${ }^{8}$ Broad Institute, Cambridge, Massachusetts, USA. ${ }^{9}$ The David H. Koch Institute for Integrative Cancer Research at Massachusetts \\ Institute of Technology, Cambridge, Massachusetts, USA. ${ }^{10}$ Dana-Farber Cancer Institute, Boston, Massachusetts, USA
}

\begin{abstract}
The mTOR pathway is a critical determinant of cell persistence and growth wherein mTOR complex 1 (mTORC1) mediates a balance between growth factor stimuli and nutrient availability. Amino acids or glucose facilitates mTORC1 activation by inducing RagA CTPase recruitment of mTORC1 to the lysosomal outer surface, enabling activation of mTOR by the Ras homolog Rheb. Thereby, RagA alters mTORC1-driven growth in times of nutrient abundance or scarcity. Here, we have evaluated differential nutrient-sensing dependence through RagA and mTORC1 in hematopoietic progenitors, which dynamically drive mature cell production, and hematopoietic stem cells (HSC), which provide a quiescent cellular reserve. In nutrient-abundant conditions, RagA-deficient HSC were functionally unimpaired and upregulated mTORC1 via nutrientinsensitive mechanisms. RagA was also dispensable for HSC function under nutritional stress conditions. Similarly, hyperactivation of RagA did not affect HSC function. In contrast, RagA deficiency markedly altered progenitor population function and mature cell output. Therefore, RagA is a molecular mechanism that distinguishes the functional attributes of reactive progenitors from a reserve stem cell pool. The indifference of HSC to nutrient sensing through RagA contributes to their molecular resilience to nutritional stress, a characteristic that is relevant to organismal viability in evolution and in modern HSC transplantation approaches.
\end{abstract}

\section{Introduction}

mTOR complex 1 (mTORC1) consists of the mTOR kinase plus multiple protein partners, including the key substrate-guiding molecule RAPTOR (1). Multiple extracellular and intracellular stimuli, such as growth factors (GFs), nutrients, and cytokines, can signal to mTORC1 (2) to upregulate anabolic metabolic processes. GF signaling leads to the removal of the inhibitory tuberous sclerosis (TSC) complex from the lysosomal surface, leading to activation of Rheb $(3,4)$. Full activation of mTORC1 then occurs by nutrients through a mechanism independent of TSC (1). Elevated levels of aa and/or glucose are sensed by multiprotein complexes on the lysosomal surface that converge on activation of a heterodimer of Rag GTPases (5-7). GTP-bound RagA or RagB dimerized with GDP-bound RagC or RagD, recruiting cytoplasmic mTORC1 via RAPTOR to the lysosome, leading to its full activation by Rheb and subsequent phosphorylation of mTORC1 substrates, such as S6K1 or 4EBP1/2 (1).

A carefully balanced level of mTORC1 activity is required for the proper functioning of the hematopoietic system, particularly under

Authorship note: D. Kalaitzidis and D. Lee contributed equally to this work. Conflict of interest: The authors have declared that no conflict of interest exists. Submitted: July 8, 2016; Accepted: January 24, 2017.

Reference information: J Clin Invest. 2017;127(4):1405-1413.

https://doi.org/10.1172/JCl89452. stress conditions (8-10). Deletion of Raptor leads to hematopoietic stem cell (HSC) failure under stress $(8,10)$, and chronic mTORC1 signaling by Tsc1 or Pten deletion can lead to HSC functional exhaustion and leukemia (10-17). Given that nutrient levels can differ markedly between homeostatic and stress conditions, particularly in the nutritional deprivation context of HSC transplant (18-20), we asked whether nutrient signaling to mTORC1 via RagA differentially affects the well-defined cell states relevant for hematopoiesis.

\section{Results}

Differential functions of RagA in homeostatic hematopoietic progenitor cell subsets. To assess the role of aa sensing in hematopoiesis, we crossed Rraga ${ }^{f l / f l}$ with Mx1-Cre mice (referred to as $\mathrm{MxCre}$ ) and deleted the floxed gene with polyinosinic:polycytidylic acid (pIpC) administration. RagA (encoded by Rraga) is the major sensor of aa and/or glucose upstream of mTOR in mice $(21,22)$. Primitive hematopoietic stem/progenitor cell (HSPC) subsets were enumerated in the bone marrow (BM) and spleen of control and Rragadeleted mice based on well-established immunophenotypes (23-25). No differences in BM HSC (either Lin-CD127-Sca1 ${ }^{+}$ $\mathrm{cKit}^{+}[\mathrm{LSK}] \mathrm{CD} 34^{-}{ }^{-\mathrm{FLT}^{-}}{ }^{-}$or the highly enriched long-term HSC $\mathrm{LSKCD}^{-} 4^{-} \mathrm{FLT3}^{-}{ }^{-} \mathrm{CD} 150^{+} \mathrm{CD} 48^{-}$fraction) or short-term reconstituting cells (STRC) $\left(\mathrm{Lin}^{-} \mathrm{CD}^{-} \mathrm{Sca}^{+} \mathrm{CKit}^{+}[\mathrm{LSK}] \mathrm{CD} 34^{+} \mathrm{Flt} 3^{-}\right)$were observed in the BM of Rraga-deleted mice (Figure 1, A and B, and Supplemental Figure 1, G and H; supplemental material available 
A

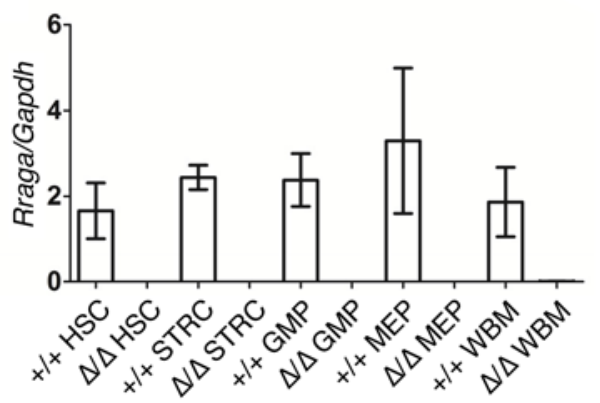

B
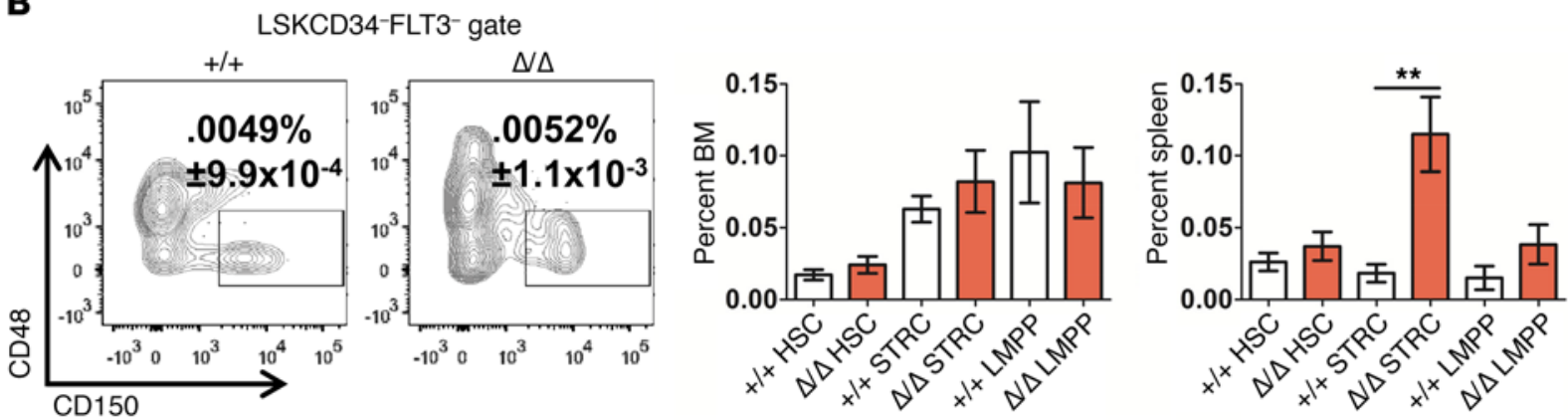

C
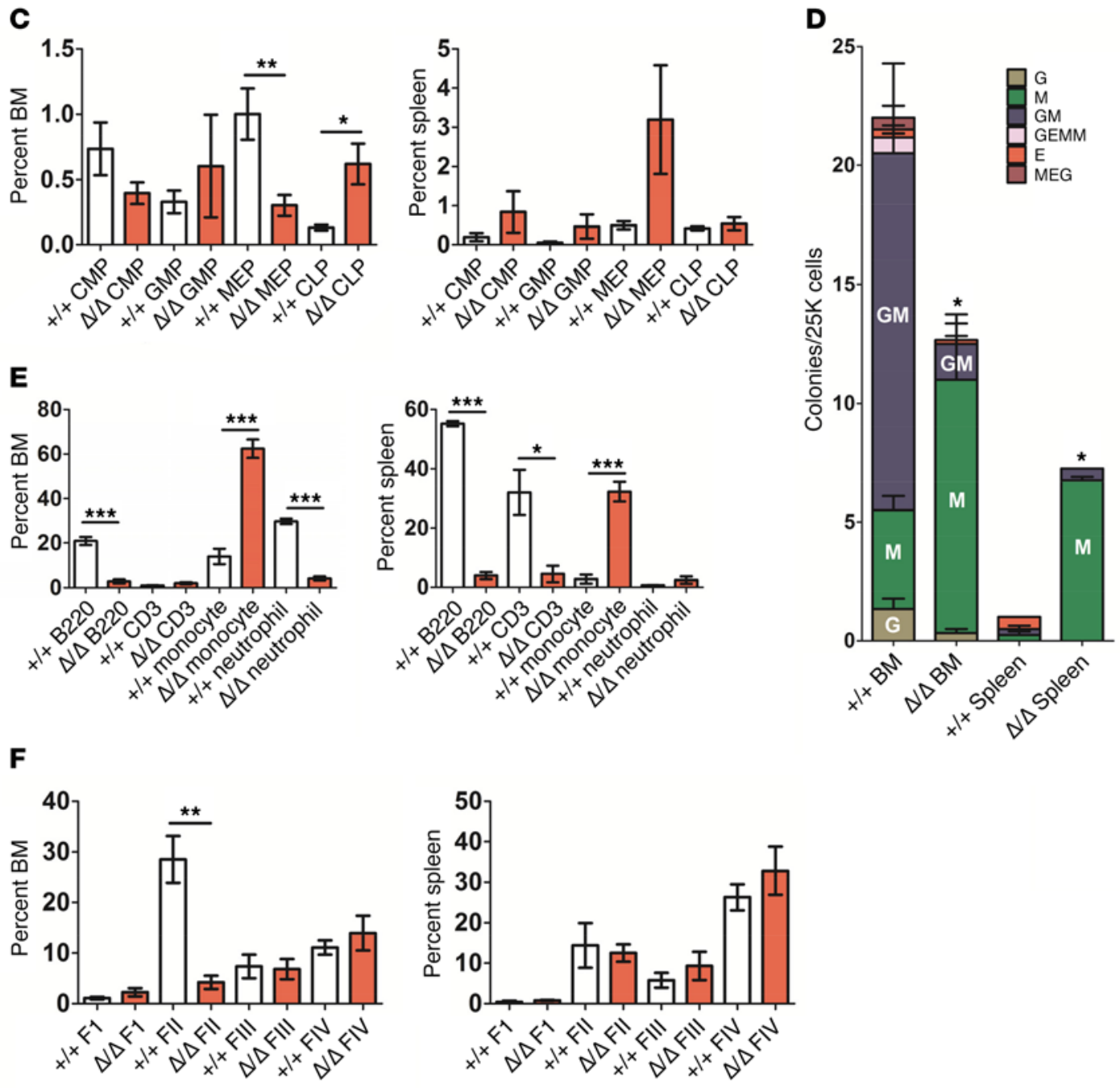
Figure 1. Rraga is required for maintaining proper progenitor differentiation and mature hematopoietic lineage cells. (A) Quantitative PCR (qPCR) on cDNA was performed to measure levels of mRNA of Rraga (normalized to Gapdh) from the indicated cell populations from mice induced with plpC 1 to 1.5 months previously (+/+, WT; $\Delta / \Delta$, induced Rraga fl/fl $M x C r e ; n=2-4)$. (B) The frequency of LSKCD34-FLT3- gated CD150+CD48- cells in BM of mice of the indicated genotypes is shown with representative FACS plot $(n=5)$. Additional HSPC populations in BM (left panel) and spleen (right panel) from the mice of the indicated genotypes 1 to 1.5 months after plpC ( $n=7$ for BM and $n=6-7$ for spleen). HSC, Lin $^{-} 7 A A D^{-}$CD127 $^{-}$Sca1 ${ }^{+} \mathrm{CKit}^{+} \mathrm{CD} 34^{-} \mathrm{FLT3}{ }^{-}$; STRC, Lin-7AAD-CD127-Sca1+CKit+CD34+FLT3-; LMPP, Lin-7AAD-CD127-Sca1+'Kit+CD34+FLT3+'. (C) The frequency of committed progenitors is shown from BM (left panel) and spleen (right panel) of mice of the indicated genotypes 1 to 1.5 months after plpC $(n=6-7)$. (D) Types and total number of colonies produced in M3434 media from cells of mice are indicated $(n=3)$. G, granulocyte; M, macrophage/monocyte; E, erythroid; GEMM, GEM-megakaryocyte; MEG, megakaryocyte-EG. (E) The frequencies of B cells (B22O), T cells (CD3), and myeloid cells (monocytes [Mac $1^{+} \mathrm{Cr} 1^{10}$ ] and granulocytes $\left[\mathrm{Mac} 1^{+} \mathrm{Gr} 1^{+}\right]$) in the BM and spleen from mice of the indicated Rraga genotype 1 to 1.5 months after deletion are shown $(n=3-4)$. (F) Ter119/CD71 staining was performed from BM and spleen to assess erythroid fractions (F1-FIV) in control and Rraga-deleted mice 1 to 1.5 months after deletion $(n=3)$. See Supplemental Figure 1 F for gating scheme. Error bars indicate SEM. ${ }^{*} P \leq 0.05 ;{ }^{* *} P \leq 0.01 ;{ }^{* *} P \leq 0.001$

online with this article; https://doi.org/10.1172/JCI89452DS1). An increase in the frequency and number of STRC was observed in the spleens of Rraga-deleted mice compared with controls (Figure $1 \mathrm{~B}$ and Supplemental Figure 1H), consistent with extramedullary hematopoiesis (EMH) (see below). We also examined the effects of Rraga loss on more downstream progenitors. While the frequency of most progenitor fractions was unaffected, Rraga deletion led to decreased megakaryocyte-erythroid progenitor cells (MEP) in the $\mathrm{BM}$ and a concomitant increase in the number of MEP in the spleen, consistent with anemia and EMH (described below) (Figure 1C and Supplemental Figure 1H). Finally, and unlike Raptordeleted progenitors, Rraga-deficient progenitors could grow ex vivo into well-defined colonies, displaying skewed differentiation, with mostly CFU-M (macrophage/monocyte) colonies produced (Figure 1D). These data indicate that aa signaling through RagA to mTORC1 does not play a role in HSC maintenance when nutrient abundance is presumably not limiting and primarily functions to influence progenitor differentiation.

Effects of Rraga on mature hematolymphoid cell subsets in homeostasis. We then examined the effects of RagA loss on mature hematopoietic and lymphoid populations. Blood cell counts were markedly affected by Rraga deletion, with decreased $\mathrm{wbc}$, rbc, and platelets (Supplemental Figure 1D). These phenotypes were indistinguishable from $\mathrm{MxCre-mediated} \mathrm{homozygous} \mathrm{deleted} \mathrm{Raptor}$ mice, though the anemia was less severe (Supplemental Figure 1D). Like constitutive $U b c-C r e-E R$-mediated deletion of Rraga and Raptor deletion, inducible and cell-restricted $\mathrm{MxCre-mediated}$ deletion also led to diminished lymphocytes (both B and splenic $\mathrm{T}$ cells) and neutrophils, with an expansion in tissue-infiltrating monocytic cells causing precipitous death (ref. 23; Figure 1E; and Supplemental Figure 1, A, B, and E). Consistent with anemia, Rraga loss also had effects on erythroid differentiation in the BM (Figure 1F and Supplemental Figure 1F). Collectively, these data indicate that Rraga controls the production/accumulation of most mature hematopoietic lineages and is critical for constraining the production of monocytes. Therefore, during steady-state hematopoiesis, nutrient signaling through RagA to mTORC1 is dispensable for stem cell maintenance, but critical for downstream progenitors in maintaining tissue homeostasis.

RagA is not required for HSC function under stress. To assess the role of RagA in HSC regeneration under stress, noninduced BM from Rragallfl $^{\text {M }} x \mathrm{Cre}^{+}$or controls was mixed at a 1:1 ratio with CD 45.1 or CD $45.1^{\text {STEM }}$ (a truly congenic mouse strain, ref. 18) and transplanted into lethally irradiated CD45.1 mice (Supplemental Figure 2A). After a 4- to 6-week engraftment phase, mice were treated with pIpC to delete RagA and chimerism was assessed (Figure 2, A and B, and Supplemental Figure 2, A and B). Rragadeficient grafts tended to give rise to lower peripheral blood (PB) chimerism over time (largely due to decreased monocytes/ neutrophils; Figure 2, A and B, and Supplemental Figure 2B). Unlike PB chimerism, Rraga $a^{f / f l} \mathrm{MxCre}$ grafts contributed as efficiently as competitor cells to the BM and spleen (Figure 2D and Supplemental Figure 2B). Chimerism in the HSC compartment and contribution to myeloid/erythroid progenitors in transplanted mice was not significantly different at approximately 19 weeks

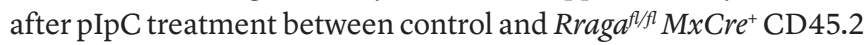
cells (Figure 2, D and E). HSPC from sorted Rragal/fll $\mathrm{Mx} \mathrm{Cre}^{+}$ CD45.2 cells contained markedly reduced Rraga mRNA expression (Figure 2F). While HSC from Rraga-deficient mice were unaffected under these stress conditions, mature cell output from Rragadeleted cells displayed cell autonomous decreases in BM B and T cells and neutrophils, with an increase in monocytic cells in the transplantation setting (Figure 2G and Supplemental Figure 2C).

We also more directly assessed whether Rraga-deficient HSC were sensitive to the acute stress of transplantation. FACS-purified Rraga-deleted HSC did not demonstrate differences compared with WT HSC in their ability to home or short-term engraft (2 weeks) when assessed in transplanted lethally irradiated mice (Supplemental Figure 2E). Finally, HSPC in Rraga-deleted mice did not show increased sensitivity to 5-FU stress (ref. 26 and Supplemental Figure 2F). Thus, Rraga, like Raptor, is required for mature hematopoietic and lymphoid output. However, unlike Raptor, Rraga appears dispensable for HSC function under the acute stress of transplantation.

Expression of Rag family members in RagA null HSPC. We hypothesized that HSC function in the absence of Rraga may not have been compromised due to a compensatory increase in Rraga expression, as described previously in Rraga-deficient mouse embryonic fibroblasts (MEFs) (21). However, Rragb expression did not increase in Rraga-deficient HSPC other than MEP (Figure 3A). Interestingly, the anemia in Rraga-deleted mice is less severe than in Raptor-deleted mice, suggesting that Rragb overexpression in response to Rraga loss may be a lineage-specific compensatory mechanism. We also examined the levels of Rragc or Rragc, either of which is required to dimerize with any Rragb expressed in Rraga null cells. Neither Rragc nor Rragc mRNA expression increased in Rraga-deleted HSPC (Figure 3A). Strikingly, both Rragc and Rragc levels were severely reduced in Rraga null HSC (Figure 3A). Thus, diminished expression of Rag family members in Rraga-deleted HSC suggest minimal Rag activity and argue against compensation by other Rag family members in HSC function. 
A

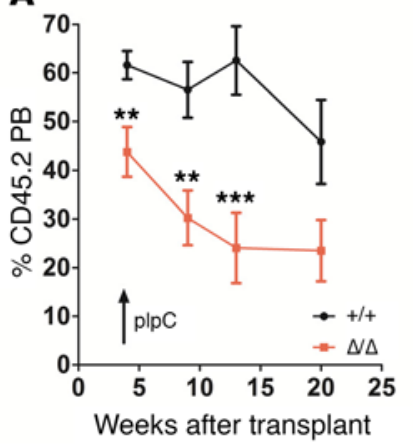

B

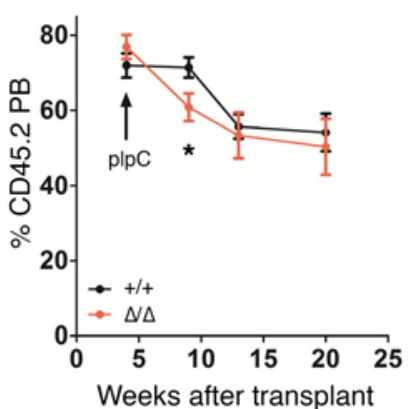

Monocyte

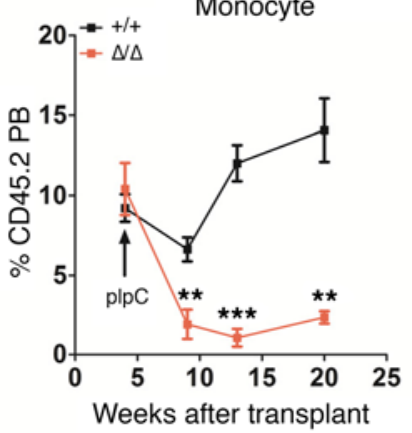

CD3
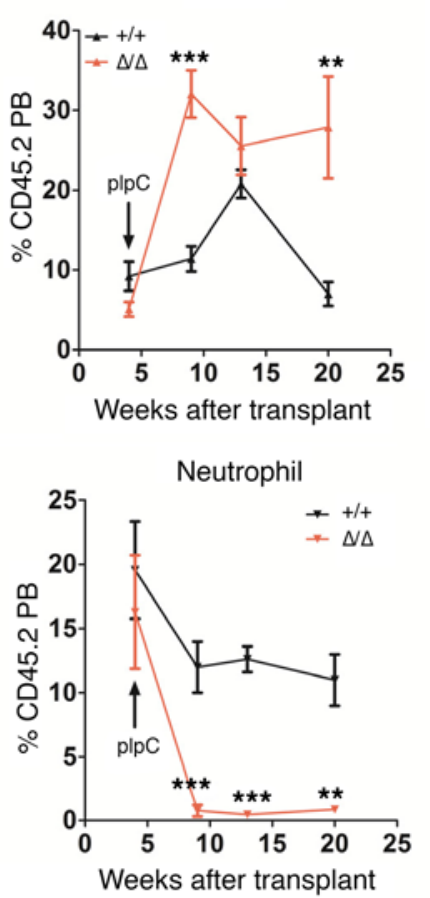

Figure 2. HSC were preserved from the Rraga-deficient setting under transplantation stress. (A) A 1:1 mixture of CD45.2 test cells (indicated) were transplanted with CD45.1 competitor cells. At the indicated time points, mice were treated with plpC and $\mathrm{PB}$ was collected and analyzed for CD45.2 chimerism $(n=4-8)$. (B) The frequencies of B cells (B220), T cells (CD3), and myeloid cells (monocytes $\left[\mathrm{Mac} 1^{+} \mathrm{Cr}^{10}\right]$ and granulocytes $\left[\mathrm{Mac}^{+} \mathrm{Cr}^{+}\right]$) from the PB of transplant recipients from $A$ are shown $(n=4-8)$. (C) BM HSC (CD48-CD150+LSK gate) chimerism was assessed 19 to 20 weeks after plpC in mice receiving cells of the indicated genotypes $(n=7)$. (D) The percentage of chimerism in each cell type of the indicated genotypes is shown at 19 to 20 weeks after plpC. HSC, Lin-Sca1+CKit ${ }^{+}$CD150+CD48- $(n=7)$; HPC, Lin ${ }^{-} \mathrm{Sca1}^{+} \mathrm{CKit}^{+} \mathrm{CD} 48^{+}(n=7)$; whole BM (WBM, $n=4)$ ). (E) The contribution to indicated progenitors from mice of the indicated genotypes was assessed in the Lin-Sca1-cKit ${ }^{+}$gate $(n=3)$. CMP, $\mathrm{Lin}^{-}{ }^{-} \mathrm{Ca1}^{-} \mathrm{CKit}^{+} \mathrm{CD} 34^{+} \mathrm{CD} 16 / 32^{+}$; CMP Lin-Sca1-CKit+CD34+CD16/32-; MEP

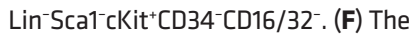
relative levels of Rraga mRNA were assessed from sorted BM CD45.2 LSK cells from mice of the indicated genotypes 20 weeks after plpC $(n=3)$. (C) The frequencies of B cells (B220), $T$ cells (CD3), and myeloid cells (monocytes $\left[\mathrm{Mac}{ }^{+} \mathrm{Cr}^{1{ }^{\circ}}\right]$ and granulocytes [Mac1+ $\left.\left.\mathrm{Cr}^{+}{ }^{+}\right]\right)$are shown at 19 to 20 weeks after plpC $(n=4)$ in the BM of mice receiving cells of the indicated genotype. Error bars indicate SEM. ${ }^{*} P \leq 0.05 ;{ }^{* *} P \leq 0.01 ;{ }^{* *} P \leq 0.001$.

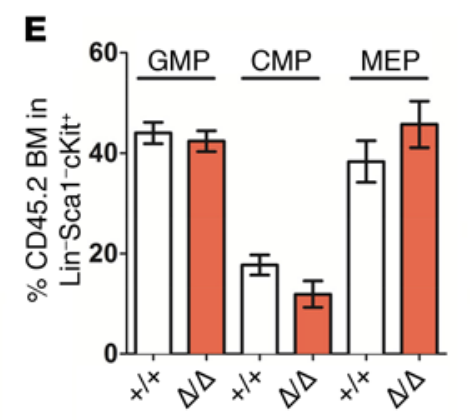

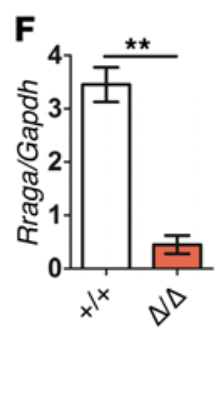

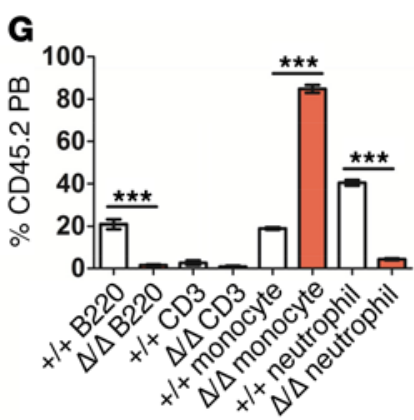

mTORC1 signaling in Rraga-deficient HSC and progenitors is insensitive to a levels. Normal HSPC responded to aa treatment by elevating levels of both p4EBP (a direct mTORC1 substrate) and pS6 (a surrogate of mTORC1 activity downstream of S6K) at both 10 and 60 minutes after aa addition (Figure 3B and Supplemental Figure 3A). HSPC were then treated with aa to assess the effects of Rraga loss on mTORC1 activity after aa deprivation (0 minutes aa treatment; Figure 3, B and C). Rraga null HSPC did not demonstrate elevation of mTORC1 activity after acute (10 minutes) aa treatment (Figure 3, C and D, and Supplemental Figure 3A); neither did they achieve localization of mTOR to the lysosomal surface comparable to that in control cells (Supplemental Figure 3B). These effects occurred despite the presence of Rragb, further suggesting that there was no compensation by Rragb. However, Rraga-deficient HSC and STRC showed elevated basal levels of p4EBP after aa deprivation (Figure 3, C and D). MEFs and heart and muscle cells that have adapted to Rraga or Rraga/b loss have previously been described as having increased 
A
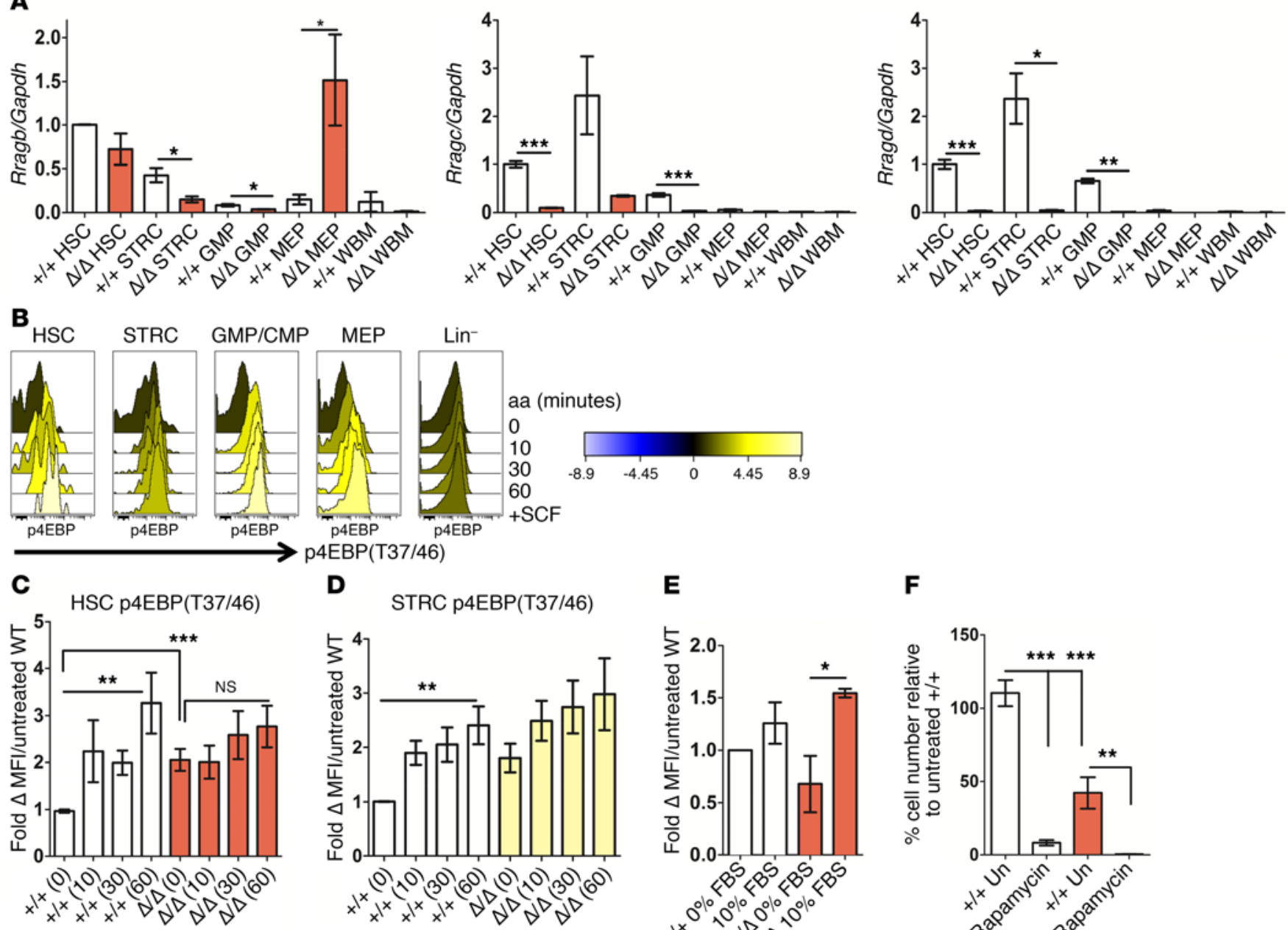

D
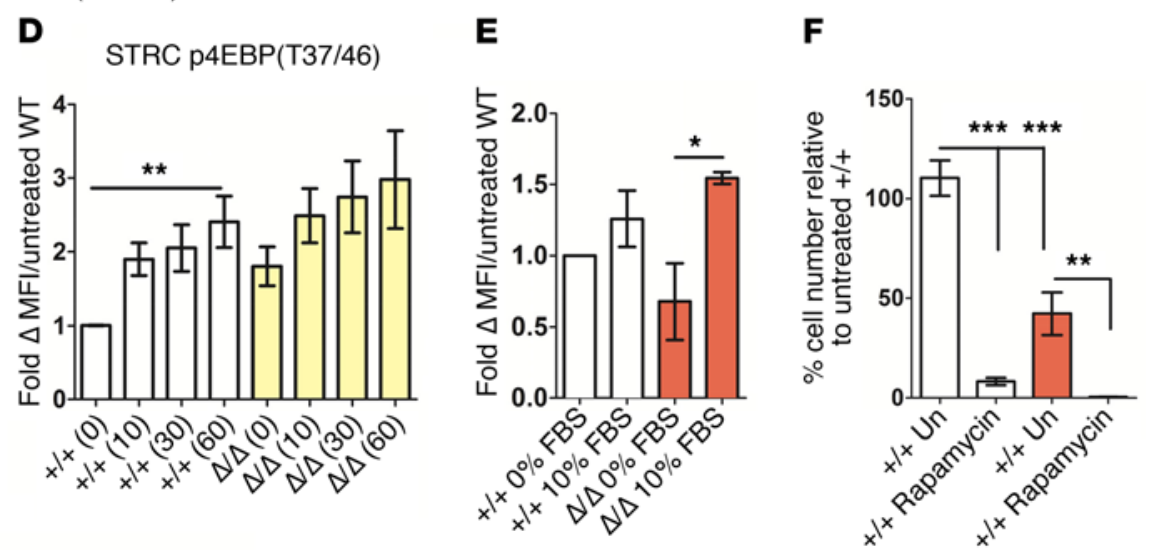

Figure 3. HSPC upregulate mTORC1 activity in the absence of Rraga, allowing cell production under stress conditions. (A) qPCR analysis of Rragb, Rragc, and Rragd levels in the indicated populations from indicated genotypes $(n=2-4)$. Level of each molecule normalized to Gapdh is shown in relation to the level in WT HSC. (B) Quantitative histograms from WT BM treated with aa for the indicated time points (minutes) showing levels of p4EBP ${ }^{37 / 46}$. Stimulation with the cytokine SCF is also shown for comparison. Color key shows fold change to untreated cells. (C and D) Lineage-depleted (Lin ${ }^{\mathrm{Dep}}$ ) BM from mice of the indicated Rraga genotypes were placed in aa-free media containing 10\% dialyzed FBS (aa depleted) for 1 hour and then stimulated with aa for the indicated time points in minutes. Intracellular flow cytometry was performed to assess levels of $\mathrm{p}^{\mathrm{E} B \mathrm{BP}^{\mathrm{T}} 3 / 46}$ in the indicated gated populations after aa stimulation for the indicated time points in minutes. The fold change in levels of p4EBP ${ }^{T 37 / 46}$ relative to WT unstimulated cells is indicated for Lin ${ }^{\text {Dep }}$ HSC and STRC $(n=5-7)$. Student's $t$ test was applied comparing time 0 values between cells of WT and homozygous knockout genotypes. Ordinary 1-way ANOVA was applied to assess differences in mean fluorescence intensity (MFI) within WT or homozygous knockout groups. (E) Lin ${ }^{\text {Dep }}$ BM from mice of the indicated Rraga genotypes was placed in aa-free media containing $0 \%$ or $10 \%$ dialyzed FBS (aa depleted). Shown are p4EBP ${ }^{\text {T37/46 }}$ levels in the HSC gate $(n=3)$. (F) LSKCD48$\mathrm{CD} 150^{+}$cells from mice of the indicated genotypes were cultured in media containing $10 \% \mathrm{FBS}$, IL-3, SCF, and TPO-containing media for 7 to 9 days with or without $10 \mathrm{nM}$ rapamycin, and cell number was assessed by CountBright Absolute Counting Beads and normalized to WT untreated ( $n=2$ independent experiments and 4 total measurements per genotype and treatment). Un, unstimulated. One-way ANOVA with Tukey's multiple comparison test was applied, and relevant statistically significant differences are noted. Error bars indicate SEM. ${ }^{*} P \leq 0.05 ;{ }^{* *} P \leq 0.01 ;{ }^{* *} P \leq 0.001$.

mTORC1 activity (21, 27). Further, mTORC1 activity in Rragadeficient, but not control, HSC was particularly sensitive to dialyzed (aa depleted) serum stimulation that was mTOR inhibitor sensitive (Figure 3E and data not shown). Basal levels of both pERK (T202/ Y204) in STRC and GMP/CMP and pAKT (S473) in HSC were also elevated in certain Rraga null HSPC populations (Supplemental Figure 3, C and D). These data suggest a general increase in GF/serum factor signaling in the absence of aa signaling through RagA to mTORC1. To test the functional consequence of elevated mTORC1 activity in Rraga null HSPC, HSC were plated in growth media with and without rapamycin (Figure 3F). Rraga null HSC could grow ex vivo, albeit at a reduced level compared with controls, and rapamycin treatment led to a marked reduction in cell number (Figure 3F). Finally, neither changes in cell cycle $(26,28)$, ROS levels (28), nor mitochondrial membrane potential (28) could explain Rragadeficient HSC phenotypes (Supplemental Figure 3, E-G). We conclude that the absence of a proper aa signal from RagA to mTORC1 leads to compensatory upregulation of basal mTORC1 activity, which is sensitive to serum factors that sustain growth under stress conditions.

Hyperactivation of RagA does not alter HSC function. Physiological levels of mTORC1 signaling are critical for proper HSC function, particularly under stress conditions. Raptor and Mtor KO HSC, 
A

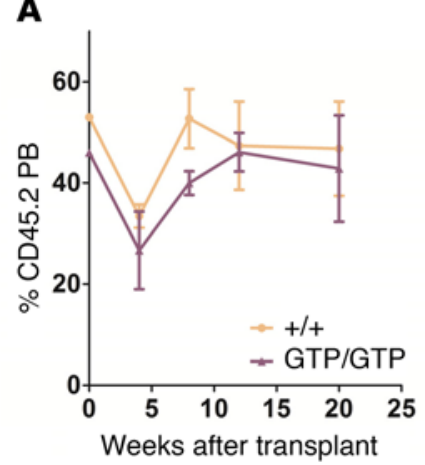

D

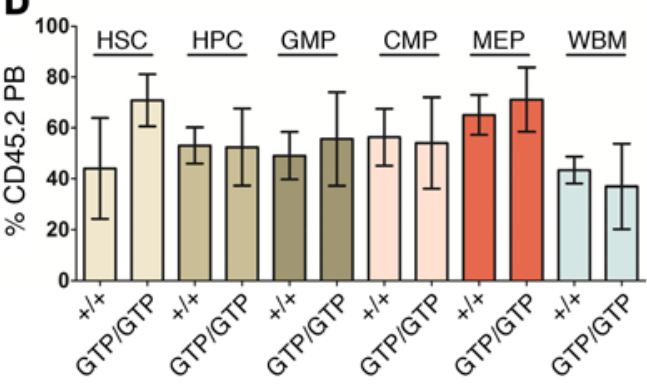

$\mathbf{F}$

STRC p4EBP(T37/46)

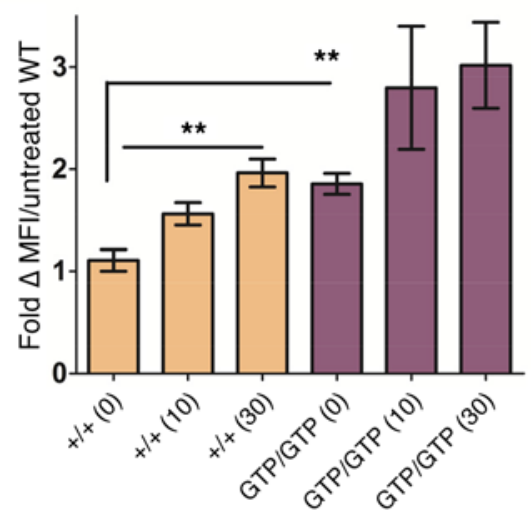

B

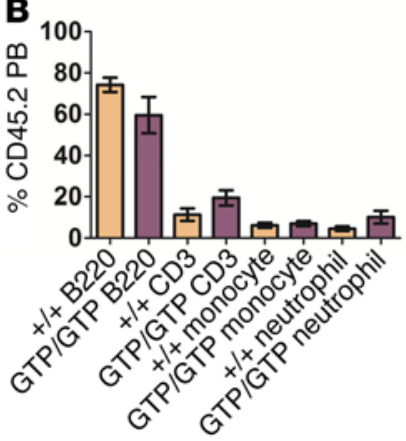

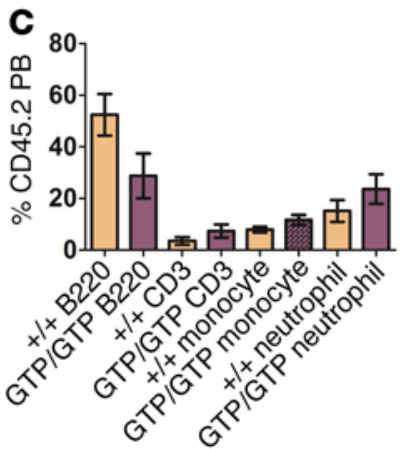

E

HSC

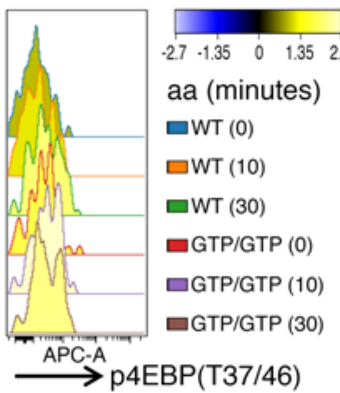

GMP/CMP p4EBP(T37/46)

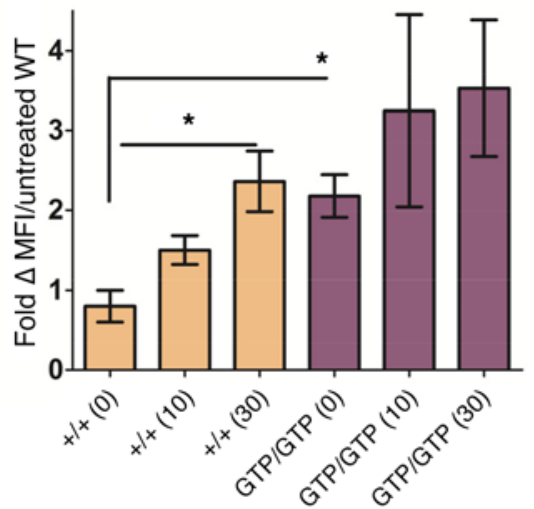

HSC P4EBP(T37/46)

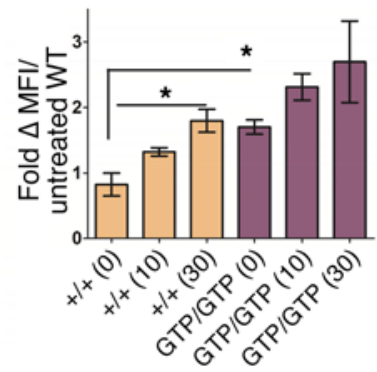

H

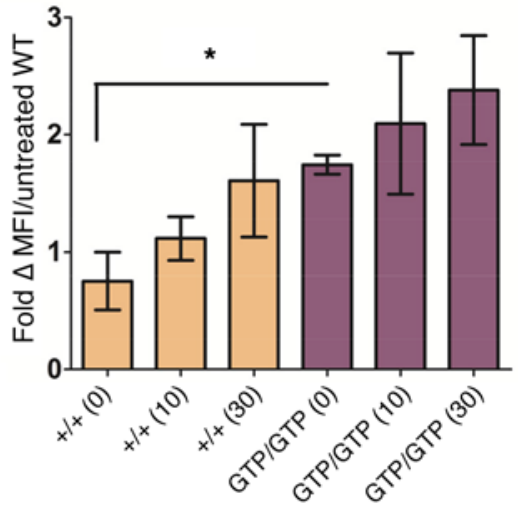

Figure 4. Constitutive nutrient signaling to mTORC1 preserves HSC function. (A) Contribution of CD45.2 cells to total PB enucleated cells from donors of the indicated genotype over time $(n=4-5)$. (B) The percentage of CD45.2 cells that are B cells (B220), T cells (CD3), and myeloid cells (monocytes [Mac1+Cr1 $\left.{ }^{10}\right]$ and granulocytes [Mac1 ${ }^{+}\left[r^{+}\right]$) in the PB approximately 20 weeks after transplantation is indicated $(n=4-5)$. (C) Analysis as in B for BM lineage approximately 28 weeks after transplantation $(n=3)$. (D) The percentage of chimerism in each cell type of the indicated genotypes is shown at approximately 28

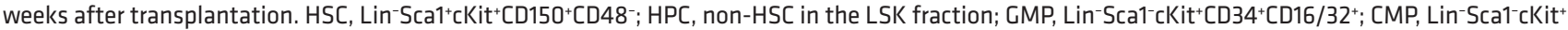
CD34+CD16/32-; MEP, Lin-Sca1-CKit+CD34-CD16/32- $(n=4)$. (E-H) Representative histograms from signal transduction experiments performed with cells from transplanted mice of the indicated genotypes. Cells were starved of aa (aa deprivation) and then stimulated for the indicated time points in minutes. Fold change above unstimulated WT cells is indicated. Lineage depleted ( in $^{\text {Dep }}$ BM from mice transplanted with cells from the indicated genotypes approximately 28 weeks after transplantation was stimulated with aa for the indicated time points (in minutes), then processed for flow cytometry. The fold

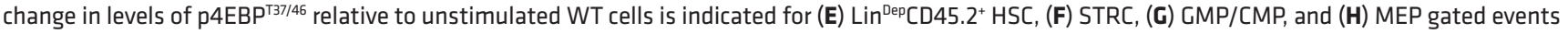
( $n=3$ mice each of the indicated genotype). Student's $t$ test was applied comparing time 0 values between cells of WT and GTP/GTP genotypes. Ordinary 1-way ANOVA was applied to assess differences in MFI within WT or homozygous knockout groups. Error bars indicate SEM. ${ }^{*} P \leq 0.05 ;{ }^{* *} P \leq 0.01$.

defective for mTOR signaling, have regeneration defects, as do HSC with constitutively active mTOR signaling driven by hyperactivation of downstream GF-responsive signaling (Pten or Tsc1 homozygous deletion) $(10,12,13,16,29)$. To assess the role of deregulation of the aa signaling arm of the mTORC1 pathway, fetal liver cells from Rraga $^{\mathrm{Q} 66 \mathrm{~L}}$ knockin mice were utilized (Figure 4A, Supplemental Fig- ure 4A, and ref. 22). Tissues from these homozygous mice (referred to as $\operatorname{RagA}^{\mathrm{GTP} / \mathrm{GTP}}$ ) are constitutively active for mTORC1 activity regardless of aa availability, due to diminished GTPase activity of this mutant Rraga $(22,29)$. Transplantation of fetal liver cells was performed, since RagA ${ }^{\text {GTP/GTP }}$ neonatal animals die prematurely. Transplantation of Pten-deficient neonatal HSC leads to normal 
A Homeostasis

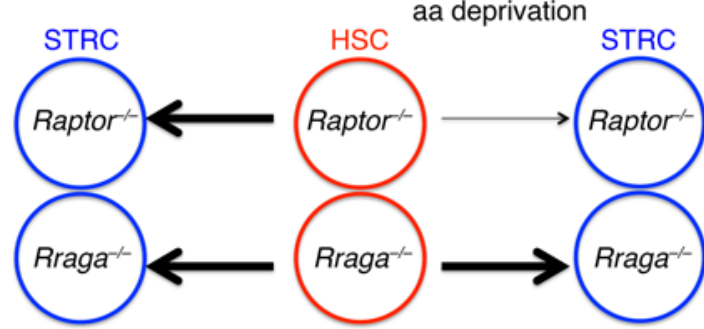

B Homeostasis,

$$
\text { Stress, or }
$$

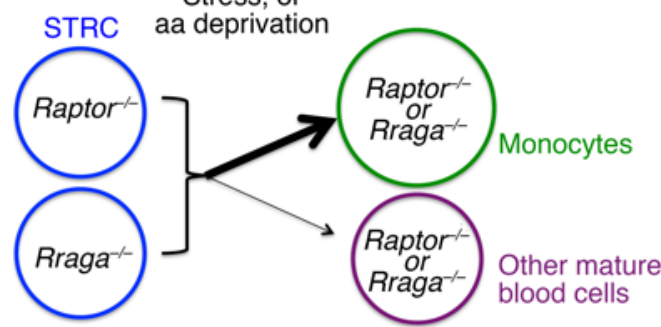

Figure 5. Model depicting differential requirements for mTORC1 regulation by nutrient signaling through RagA in homeostatic versus stress hematopoiesis. (A) Under homeostatic conditions, HSC do not depend on mTORC1. Under hematopoietic stress, mTORC1 is critical for HSC function, as previously shown by Raptor deletion (Raptor ${ }^{-1-}$ ). However, nutrient sensing by RagA is not required for mTORC1 activity in HSC and Rraga ${ }^{-1-}$ HSC are indifferent to aa levels. (B) In contrast, progenitors (STRC) depend on RagA-regulated mTORC1 activation to maintain normal production of mature blood cells. Distinct effects are noted on monocyte production (increased) compared with other blood cells (decreased) with either Raptor or Rraga deletion.

reconstitution in recipients up until the time of transition from the fetal to adult HSC program, at which point hematopoiesis from Pten-deleted HSC declines (15). Thus, our system would allow us to monitor effects of RagA hyperactivation in both fetal and adult HSC.

Fetal liver cells from controls and RagA ${ }^{\mathrm{GTP} / G T P}$ mice were then competitively transplanted versus CD $45.1^{\text {STEM }}$ whole BM cells (Figure $4 \mathrm{~A}$ and Supplemental Figure 4A). RagA ${ }^{\text {GTP/GTP }}$ had no detectable effect on chimerism, the number of HSPC or mature hematopoietic cell types, or on gross hematopoietic parameters up to 28 weeks after transplantation. We confirmed the presence of recombined alleles after long-term transplantation (Figure 4, B-D, and Supplemental Figure 4). RagA ${ }^{\mathrm{GTP} / \mathrm{GTP}}$ HSC in transplanted mice retained elevated basal mTORC1 signaling as well as increased levels of p4EBP after 10 minutes of aa stimulation for at least 28 weeks (Figure 4E). Other RagA ${ }^{\text {GTP/GTP }}$ HSPC populations also had elevated basal mTORC1 signaling compared with control cells (Figure 4, F-H). Unlike mutations that mimic hyper-GF signaling, such as deletion of Pten or Tsc1, hyperactivation of nutrient sensing by activating Rraga mutations do not perturb HSC function under the stress of transplantation.

\section{Discussion}

In this study, we demonstrate that HSC are unaffected in the BM and STRC are increased in the spleen by the loss of Rraga under steadystate conditions. This is consistent with previous data from us and others regarding mTORC1 based on Raptor deletion (8-10). Notably, we demonstrate that the previously demonstrated need for mTORC1 in HSC regeneration under stress is Rraga independent. However, Rraga is necessary for the previously defined mTORC1-regulated levels of rbc, platelets, and wbc in the blood and for differentiation decisions along the neutrophil/monocyte lineage. Thus, nutrient sensing by RagA and its activation of mTORC1 play different roles in steady-state compared with stress hematopoiesis and in HSC compared with more mature progenitors (Figure 5).

The leukopenia, anemia, and thrombocytopenia with rapamycin analog therapy in patients may reflect nutrient-sensing aspects of mTORC1 regulation (30). This possibility is supported by the observation that $\mathrm{L}$-leucine can regulate hemoglobin levels through an mTORC1/4EBP1 mechanism (31). The effect of nutrient signaling through $\mathrm{mTORC} 1$ on differentiation is evident in the preferential production of monocytes seen with Raptor, Rraga, or folliculin (a GTPase-activating protein for Rragc/Rragd, ref. 32) deletion or deprivation of glutamine $(10,21,33,34)$. These data indicate that nutrient signaling through $\mathrm{mTORC} 1$ alters mature cell production and may play an instructive rather than simply a permissive role in lineage-fate decisions. During nutritional stress, progenitors enhance generation of a fundamental cell of innate host defense at the expense of other hematopoietic cell types.

However, in more immature HSPC, loss of the aa-sensing dimension of mTORC1 signaling is without significant cost to cell number or function under stress, likely due to upregulation of mTORC1 independently of RagA. This upregulation could be particularly important when aa levels are low. For example, it may explain why patients undergoing HSC transplantation rarely experience graft failure, despite being in an extreme catabolic and poorly nourished state $(19,20,35)$. Abundance of nutrients also leaves HSC unaffected, as demonstrated by RagA ${ }^{\mathrm{GTP} / \mathrm{GTP}}$, providing no competitive advantage or disadvantage to HSC. These data also show that increased signaling to mTORC1, per se, is not deleterious to HSC function. While mutations that mimic chronic and less regulated mTORC1 activation by GFs, such as Pten or Tsc1 deletion, lead to HSC impairment and sometimes leukemia $(11-14,16,17)$, genetic upregulation of mTORC1 activity through nutrient signaling does not impair HSC function. It appears then that HSC enact an mTORC1-signaling process that protects them from both variable nutrient availability and oncogenesis due to excess nutrient stimulation.

The insensitivity of HSC to RagA signaling has implications for the development of pharmacological mTORC1 inhibitors in metabolic diseases and cancer. To date, specific mTORC1 inhibitors that do not affect mTORC2 (which plays distinct roles in human health) have not been identified. Identification of drugs that affect nutrient signaling via RagA to mTORC1 would result in specific mTORC1 inhibitors that would not affect mTORC2. Our data suggest that inhibiting nutrient signaling to mTORC1 via RagA would not impair HSC even under stress conditions. Thus, a pharmacological inhibitor of nutrient signaling to mTORC1 as opposed to a general mTOR inhibitor would potentially have fewer long-term hematological side effects, particularly when used in combination with chemotherapy or radiation.

In summary, HSC are essentially indifferent to the decreased or increased activities of the aa-sensing arm of mTORC1 signaling and perhaps thereby, from the vicissitudes of nutrient availability. In contrast, more mature hematopoietic populations are highly affected by changes in aa and glucose-sensing RagA activity. These distinctions correlate with the different functions of the 
stem and progenitor populations with the more nutrient-insensate stem cell population, providing the deep reserve of hematopoietic tissue that must be resilient to varying environments and stress conditions to preserve organismal viability. The differential governance of mTORC1 through RagA is one specific molecular basis for accomplishing this distinct function of the stem cell pool.

\section{Methods}

Mouse and animal procedures. All mice were kept in a specific pathogenfree facility at Massachusetts General Hospital. Rragat/fl , described previously (21), were backcrossed to at least 6 generations in the C57BL/6 and crossed with Mx1-Cre mice (36). Raptorl/fl Mx1-Cre mice, Rraga $^{\mathrm{Q} 66 \mathrm{~L}}\left(\mathrm{RagA}^{\mathrm{GTP} / \mathrm{GTP}}\right)$ mice, and $\mathrm{pIpC}$ administration were described previously $(10,22)$. CD $45.1^{\text {STEM }}$ mice (CD $45.1^{*}$, a truly congenic mouse strain) have been previously described (18). Transplantations were performed as described previously (10).

Flow cytometry. Flow cytometry was performed as described previously with antibodies listed in Supplemental Methods and Materials (10).

aa signaling and intracellular flow cytometry. Intracellular flow cytometry was performed with modifications to previously described protocols $(10,37)$. BM was isolated by crushing long bones and sternum in $0.5 \% \mathrm{BSA} / \mathrm{PBS}$, rbc lysed, and cells passed through $40-\mu \mathrm{m}$ or $70-\mu \mathrm{m}$ nylon cell strainers (BD). To ultimately exclude dead cells from the analysis, $1 \mathrm{ml}$ of BM solution was incubated with $1 \mu \mathrm{l}$ of fixable viability dye eFluor 780 (eBioscience) on ice for 10 minutes, washed twice, and resuspended in 1 $\mathrm{ml}$ of $0.5 \% \mathrm{BSA} / \mathrm{PBS}$. BM was then depleted of mature cell lineages by incubating cells at room temperature for 15 minutes with biotin-conjugated antibodies to the following: CD3e (clone 145-2c11), CD4 (clone GK1.5), CD8a (clone 53-6.7), CD19 (clone ID3), B220 (clone RA3-6B2), GR1 (clone RB6-8C5), TER119 (clone TER-119), and CD127 (clone A7R34). Then $50 \mu$ l of EasySep Streptavidin RapidSpheres (Stem Cell Technologies) was added and solution incubated at room temperature for 2 to 5 minutes before separation of cells from magnetic beads using an EasySep Magnet.

$\mathrm{BM}$ was then incubated at $37^{\circ} \mathrm{C}$ in aa-free RPMI media (US Biological) containing 10\% FBS (Life Technologies) that was dialyzed using a 3500 MW cutoff Slide-A-Lyzer (Thermo Fisher Scientific) for approximately 1 hour. Cells were then stimulated with a $1 \times$ mixture of aa (Sigma-Aldrich) supplemented with L-glutamine (300 $\mathrm{mg} / \mathrm{l}$ final) (Thermo Fisher Scientific) for indicated time points. Cells were then fixed with paraformaldehyde (Electron Microscopy Sciences) for 10 minutes at room temperature at a final concentration of $1.5 \%$, permeabilized with ice-cold acetone for 10 minutes on ice, washed, and stained for flow cytometry. Cells were typically stained with BV605 SCA1 (clone D7), BUV395 cKIT (clone 2B8), eFluor450 CD34 (clone RAM34), Ax647 p4EBP (clone 236B4), and PE pS6 (clone N4-41). PE-CF594 pERK (clone 20A), Ax488 pAKT (clone 193H12), FITC CD45.2 (clone 104), and PE CD45.1 (clone A20) were also used in some analyses. Data were analyzed either using FlowJo (Treestar) or Cytobank software (38).

Gene expression. RNA/cDNA isolation and syntheses were performed as described previously (10). The following TaqMan assayswere used:Rraga, Mm02525632_s1; Rragb, Mm01348407_m1; Rragc, Mm00600306 m1; Rragd, Mm00546741_m1; and Gapdh Mm99999915_g1 (Applied Biosystems). Reactions were run on an Applied Biosystems StepOnePlus qPCR machine. Since Rragb RNA can be difficult to detect in normal cells and/or tissue, a standard curve was established using cDNA prepared from Rraga null MEFs, which express high levels of Rragb (21).

Statistics. Sample size required for the experiments was estimated based upon results of preliminary data. No blinding or randomization was performed for any of the experiments. The statistical significance of differences between data points was assessed by either 2-tailed unpaired Student's $t$ test or 1-way ANOVA with or without Tukey's multiple comparison test as implemented in Graphpad Prism software and as indicated. Kaplan-Meier log-rank test was used to analyze mouse survival data.

Study approval. All mice were kept in a specific pathogen-free facility at Massachusetts General Hospital. All mouse studies and breeding were carried out under the approval of the Institutional Animal Care and Use Committee of Massachusetts General Hospital.

\section{Author contributions}

DK, DL, and DTS designed research, analyzed data, and wrote the manuscript. DK and DL carried out most of the experimental work with the help of AE, NN, AP, NB, and GDV. DBS and FEM generated and provided CD45.1 $1^{\text {STEM }}$ mice. DMS provided Rraga $a^{f / f l}$ and $\mathrm{RagA}^{\mathrm{GTP} / \mathrm{GTP}}$ mice. YK designed and performed confocal microscopy experiments. DN provided statistical expertise. DTS directed research and wrote the manuscript.

\section{Acknowledgments}

We thank Jacqueline Bachand for expert technical assistance and Rushdia Yusuf and Borja Saez for experimental advice. We thank Massachusetts General Hospital/Harvard Stem Cell Institute Center for Regenerative Medicine flow cytometry core (A. Galvin and M. Handley). DK was supported by NIH National Institute of Diabetes and Digestive and Kidney Diseases (NIDDK) award K01DK092300. DL was supported by the Massachusetts General Hospital Fund for Medical Discovery fellowship. YK was supported by the Dubai Harvard Foundation for Medical Research. FEM was funded by a clinician-scientist training award from the Canadian Institutes of Health Research. GDV was supported by NIH grant DP5OD012146. DTS and DL were supported by NIH National Heart, Lung, and Blood Institute (NHLBI) grants U01HL100402, HL97794, and HL044851. DMS was supported by NIH grant R01CA129105, CA103866, AI047389, and R21AG042876. The data reported in this manuscript are tabulated in the main paper and in the Supplemental Methods and Materials.

Address correspondence to: Demetrios Kalaitzidis, CRISPR Therapeutics, 610 Main Street, Cambridge, Massachusetts 02139, USA. Phone: 617.315.4600; E-mail: d.kalaitzidis@crisprtx.com. Or to: David T. Scadden, 185 Cambridge Street, Boston, Massachusetts 02114, USA. Phone: 617.726.5615; E-mail: david_scadden@harvard.edu.

D. Kalaitzidis's present address is: CRISPR Therapeutics, Cambridge, Massachusetts, USA.

A. Efeyan's present address is: Spanish National Cancer Research Center (CNIO), Madrid, Spain.

G.D. Victora's present address is: Laboratory of Lymphocyte Dynamics, The Rockefeller University, New York, New York, USA. 
1. Bar-Peled L, Sabatini DM. Regulation of mTORC1 by amino acids. Trends Cell Biol. 2014;24(7):400-406.

2. Dibble CC, Manning BD. Signal integration by mTORC1 coordinates nutrient input with biosynthetic output. Nat Cell Biol. 2013;15(6):555-564.

3. Demetriades C, Doumpas N, Teleman AA. Regulation of TORC1 in response to amino acid starvation via lysosomal recruitment of TSC2. Cell. 2014;156(4):786-799.

4. Menon S, et al. Spatial control of the TSC complex integrates insulin and nutrient regulation of mTORC1 at the lysosome. Cell. 2014;156(4):771-785.

5. Kim E, Goraksha-Hicks P, Li L, Neufeld TP, Guan KL. Regulation of TORC1 by Rag GTPases in nutrient response. Nat Cell Biol. 2008;10(8):935-945.

6. Sancak Y, Bar-Peled L, Zoncu R, Markhard AL, Nada S, Sabatini DM. Ragulator-Rag complex targets mTORC1 to the lysosomal surface and is necessary for its activation by amino acids. Cell. 2010;141(2):290-303

7. Sancak Y, et al. The Rag GTPases bind raptor and mediate amino acid signaling to mTORC1. Science. 2008;320(5882):1496-1501.

8. Guo F, et al. Mouse gene targeting reveals an essential role of mTOR in hematopoietic stem cell engraftment and hematopoiesis. Haematologica. 2013;98(9):1353-1358.

9. Hoshii T, et al. mTORC1 is essential for leukemia propagation but not stem cell self-renewal. J Clin Invest. 2012;122(6):2114-2129.

10. Kalaitzidis D, et al. mTOR complex 1 plays critical roles in hematopoiesis and Pten-loss-evoked leukemogenesis. Cell Stem Cell. 2012;11(3):429-439.

11. Campbell TB, Basu S, Hangoc G, Tao W, Broxmeyer HE. Overexpression of Rheb2 enhances mouse hematopoietic progenitor cell growth while impairing stem cell repopulation. Blood. 2009;114(16):3392-3401.

12. Chen C, et al. TSC-mTOR maintains quiescence and function of hematopoietic stem cells by repressing mitochondrial biogenesis and reactive oxygen species. J Exp Med. 2008;205(10):2397-2408.

13. Gan B, et al. mTORC1-dependent and -independent regulation of stem cell renewal, differentia- tion, and mobilization. Proc Natl Acad Sci U S A. 2008;105(49):19384-19389.

14. Kharas MG, et al. Constitutively active AKT depletes hematopoietic stem cells and induces leukemia in mice. Blood. 2010;115(7):1406-1415.

15. Magee JA, Ikenoue T, Nakada D, Lee JY, Guan KL, Morrison SJ. Temporal changes in PTEN and mTORC2 regulation of hematopoietic stem cell self-renewal and leukemia suppression. Cell Stem Cell. 2012;11(3):415-428.

16. Yilmaz OH, et al. Pten dependence distinguishes haematopoietic stem cells from leukaemiainitiating cells. Nature. 2006;441(7092):475-482.

17. Zhang J, et al. PTEN maintains haematopoietic stem cells and acts in lineage choice and leukaemia prevention. Nature. 2006;441(7092):518-522.

18. Herrmann VM, Petruska PJ. Nutrition support in bone marrow transplant recipients. Nutr Clin Pract. 1993;8(1):19-27.

19. Lenssen P, Bruemmer B, Aker SN, McDonald GB. Nutrient support in hematopoietic cell transplantation. JPEN J Parenter Enteral Nutr. 2001;25(4):219-228.

20. Thompson JL, Duffy J. Nutrition support challenges in hematopoietic stem cell transplant patients. Nutr Clin Pract. 2008;23(5):533-546.

21. Efeyan A, et al. RagA, but not RagB, is essential for embryonic development and adult mice. Dev Cell. 2014;29(3):321-329.

22. Efeyan A, et al. Regulation of mTORC1 by the Rag GTPases is necessary for neonatal autophagy and survival. Nature. 2013;493(7434):679-683.

23. Kiel MJ, Yilmaz OH, Iwashita T, Yilmaz OH, Terhorst C, Morrison SJ. SLAM family receptors distinguish hematopoietic stem and progenitor cells and reveal endothelial niches for stem cells. Cell. 2005;121(7):1109-1121.

24. Osawa M, Hanada K, Hamada H, Nakauchi H. Long-term lymphohematopoietic reconstitution by a single CD34-low/negative hematopoietic stem cell. Science. 1996;273(5272):242-245.

25. Yamamoto R, et al. Clonal analysis unveils self-renewing lineage-restricted progenitors generated directly from hematopoietic stem cells. Cell. 2013;154(5):1112-1126.

26. Goncalves KA, et al. Angiogenin promotes hematopoietic regeneration by dichotomously regulating quiescence of stem and progenitor cells. Cell.
2016;166(4):894-906.

27. Kim YC, et al. Rag GTPases are cardioprotective by regulating lysosomal function. Nat Commun. 2014;5:4241.

28. Wang YH, et al. Cell-state-specific metabolic dependency in hematopoiesis and leukemogenesis. Cell. 2014;158(6):1309-1323.

29. Hirose E, Nakashima N, Sekiguchi T, Nishimoto T. RagA is a functional homologue of S. cerevisiae Gtr1p involved in the Ran/Gsp1-GTPase pathway. JCell Sci. 1998;111(pt 1):11-21.

30. Xu J, Tian D. Hematologic toxicities associated with mTOR inhibitors temsirolimus and everolimus in cancer patients: a systematic review and meta-analysis. Curr Med Res Opin. 2014;30(1):67-74

31. Chung J, et al. The mTORC1/4E-BP pathway coordinates hemoglobin production with L-leucine availability. Sci Signal. 2015;8(372):ra34.

32. Tsun ZY, et al. The folliculin tumor suppressor is a GAP for the RagC/D GTPases that signal amino acid levels to mTORC1. Mol Cell. 2013;52(4):495-505.

33. Mercier FE, Sykes DB, Scadden DT. Single targeted exon mutation creates a true congenic mouse for competitive hematopoietic stem cell transplantation: the C57BL/6-CD45.1(STEM) mouse. Stem Cell Reports. 2016;6(6):985-992.

34. Oburoglu L, et al. Glucose and glutamine metabolism regulate human hematopoietic stem cell lineage specification. Cell Stem Cell. 2014;15(2):169-184

35. Zatarain L, Savani BN. The role of nutrition and effects on the cytokine milieu in allogeneic hematopoietic stem cell transplantation. Cell Immunol. 2012;276(1-2):6-9.

36. Kuhn R, Schwenk F, Aguet M, Rajewsky K. Inducible gene targeting in mice. Science. 1995;269(5229):1427-1429.

37. Kalaitzidis D, Neel BG. Flow-cytometric phosphoprotein analysis reveals agonist and temporal differences in responses of murine hematopoietic stem/progenitor cells. PLoS One. 2008;3(11):e3776.

38. Kotecha N, Krutzik PO, Irish JM. Web-based analysis and publication of flow cytometry experiments. Curr Protoc Cytom. 2010; Chapter 10:Unit10.17. 